\title{
The KePler Guest Observer Programme
}

\author{
Thomas Barclay ${ }^{1,2}$ \\ ${ }^{1}$ NASA Ames Research Center, MS 244-30, Mountain View, CA 94035, USA \\ ${ }^{2}$ Bay Area Environmental Research Institute, Sonoma, CA 95476, USA \\ email: thomas.barclay@nasa.gov
}

\begin{abstract}
The KePLER Guest Observer Office is dedicated to the service of the broad science community, with a charter to promote the exploitation of KEPLER data and broaden the scientific impact of the KEPLER mission. There are four routes by which to gain access to KEPLER data: the Guest Observer programme annual call for proposals, the quarterly call for Director's Discretionary Targets, the KEPLER Asteroseismic Science Consortium, and by accessing the KePLER public data archive. The Guest Observer Office supports all users of KePLER data no matter which route they took to access data, and to that end have developed software tools which can be used by the community in their data analyses.
\end{abstract}

Keywords. stars: variables: general, techniques: photometric, methods: data analysis

\section{The Kepler Mission}

The KEPLER spacecraft was launched in 2009 March on a mission to determine the frequency of Earth-like planets in or near the habitable zone around Sun-like stars (Koch et al. 2010; Borucki et al.] 2009; Borucki et al. 2010; Caldwell et al. 2010). The 0.95-m aperture telescope is in a heliocentric, Earth-trailing orbit and almost continually observes the same $116 \mathrm{deg}^{2}$ field of view. KEPLER thus continually observes over $1.6 \times 10^{5}$ targets at a cadence of $30 \mathrm{~min}$ with milli- to micro- magnitude precision and has announced the discovery of over 1200 planet candidates, with sizes ranging from smaller than the Earth to twice the size of Jupiter (Borucki et al. 2010; Fressin et al. submitted) with orbital periods ranging from less than a day to nearly a year (Welsh et al., submitted).

\section{Astrophysics with KePLER}

The scientific endeavours accomplished with data from the KEPLER mission encompass a multitude of fields of astrophysics research, from studies of the zodiacal dust in the Solar System (Witteborn et al. 2011) to distant AGNs (Mushotzky et al. 2011). There are four specific fields which KEPLER has the potential to revolutionise: those of asteroseismology (e.g., Bedding et al. 2011; Chaplin et al. 2011; Antoci et al. 2011; Beck et al. 2011), gyrochronology (Meibom et al. 2011), stellar activity (e.g., Basri et al. 2011; Walkowicz et al. 2011) and binary stars (e.g., Carter et al. 2011; Derekas et al. 2011; Slawson et al. 2011).

\section{Opportunities to Access KePler Data}

There are four paths for obtaining KEPLER data on specific targets of interest: the full yearly call for Guest Observer proposals, the quarterly Director's Discretionary Target programme, the KEPLER Asteroseismic Science Consortium and the KEPLER data archive. 


\subsection{The annual Guest Observer competition*}

Once a year there is a call for proposals to observe targets within the KEPLER field of view. The call is open to everyone; proposers need not be based in the United States. There are $\sim 5000$ long-cadence slots (one data point every $29.4 \mathrm{~min}$ ) and 40 short-cadence ones (one data point every $58.8 \mathrm{~s}$ ) available at any one time in this programme. The Kepler Guest Observer (GO) Office particularly solicits proposals from the astronomical community for:

(a) New sources of astrophysical interest, and

(b) Observations of existing targets on the exoplanet list for scientific investigations that are distinct from exoplanetary and related science.

\subsection{Director's Discretionary Targets}

The standard Guest Observer competition occurs on an annual cycle. Because of the proposal review time-line, associated data processing and archive activities, there is currently a 6 -month delay between proposal submission and first observations, and a 1-year wait between proposal submission and the delivery of the first Guest Observer data. To provide a faster mechanism for headline KEPLER astrophysics, the Guest Observer Office provides an alternative means for acquiring KEPLER data through the Director's Discretionary Target (DDT) programme.

The DDT program is a quarterly competition. Observations can be proposed at any time, and approved targets will be added during the next spacecraft roll. Up to 100 DDTs are available each quarter. Proposals from all institutions and countries are encouraged. The proposal process is informal, requiring an email request from the proposer to the Guest Observer Officet. The purpose of the DDT programme is to:

- Provide a fast track to KEPLER data so as to yield rapid, high-impact science and thus build a significant sample of KEPLER GO papers rapidly

- Respond to targets of GO interest, newly dropped from the planetary list. Targets can be reinstated onto the observing list via a DDT request

- Respond to "targets of opportunity"

- Permit pilot studies of small samples prior to the next GO solicitation

- Enhance existing GO programmes with additional data.

\subsection{The Kepler Asteroseismic Science Consortium $\ddagger$}

The main body of asteroseismic investigation of KEPLER data has been performed by members of the KEPLER Asteroseismic Science Consortium (KASC), a collection of over 500 scientists from all over the world who are studying stellar pulsations in order to understand the internal structure of stars. KASC members are guaranteed a minimum of 1700 long- and 140 short-cadence targets, and welcome new members who are able to contribute to the analysis of KEPLER asteroseismic data.

\subsection{Archival data}

The KEPLER archive is hosted by the Multimission Archive at the Space Telescope Sciences Institute (MAST)§. All KEPLER data are archived, and many of them are publicly available for scientific exploitation. MAST hosts not only the archived light-curve data but also the calibrated and uncalibrated pixel-level data and full-frame images which

$*$ For specific details of the call in a given year see http://keplergo.arc.nasa.gov/.

†Email: kepler-go@lists.nasa.gov

$\ddagger$ More information about the KASC is available from http://astro.phys.au.dk/KASC/.

$\S$ KEPLER data can be obtained from http://archive.stsci.edu/kepler. 
contain all pixels within the KEPLER field of view and not just those within the predefined pixel masks used for long- and short-cadence observations. In 2011 October, over $1.1 \times 10^{6}$ individual light curves for $1.7 \times 10^{5}$ sources are publicly available.

\section{Community Software*}

The Guest Observer Office has developed a suite of software called PYKE, which can be used in the processing and analysis of KEPLER data. Of particular importance are the tools KEPEXTRACT and KEPCOTREND. The light-curve products at MAST are extracted from the pixel-level data using an aperture which optimises signal to noise - this product contains significant instrumental systematics. The KEPEXTRACT tool allows the user to chose different apertures. The reasons for doing that include:

- Using all pixels in the aperture

- Creating a light curve for certain sources, since the KEPEXTRACT pipeline does not produce a light curve for sources observed with custom or dedicated pixel masks

- Constructing pixel light curves in which the time series for a single pixel can be examined

- Obtaining light curves for extended sources which may be poorly sampled by the optimal aperture.

Astrophysical variability among the KEPLER targets is expected to be uncorrelated, so any apparent correlation is presumed to be due to instrumental systematics. Such correlations can be represented as a linear combination of orthogonal vectors known as cotrending basis vectors. The KEPLER project has released a product containing the cotrending basis vectors and has made them available at MAST $\dagger$.

The Guest Observer Office has developed a tool called KEPCOTREND for fitting cotrending basis vectors to a light curve. The fitted instrumental signal can then be subtract from the time series, to leave a clean light curve.

\section{References}

Antoci, V., et al. 2011, Nature, 477, 570

Basri, G., et al. 2011, AJ, 141, 20

Beck, P. G., et al. 2011, Science, 332, 205

Bedding, T. R., et al. 2011, Nature, 471, 608

Borucki, W., et al. 2009, in: F. Pont, D. Sasselov \& M. Holman (eds.), Transiting Planets, (IAU S 253) (Cambridge: Cambridge University Press), p. 289

Borucki, W. J., et al. 2010, Science, 327, 977

Caldwell, D. A., et al. 2010, ApJ (Letters), 713, L92

Carter, J. A., et al. 2011, Science, 331, 562

Chaplin, W. J., et al. 2011, Science, 332, 213

Derekas, A., et al. 2011, Science, 332, 216

Koch, D. G., et al. 2010, ApJ (Letters), 713, L79

Meibom, S., et al. 2011, ApJ (Letters), 733, L9

Mushotzky, R. F., Edelson, R., Baumgartner, W. H., \& Gandhi, P. 2011, arXiv:1111.0672

Slawson, R. W., et al. 2011, AJ, 142, 160

Walkowicz, L. M., et al. 2011, AJ, 141, 50

Witteborn, F. C., Van Cleve, J. E., Borucki, W., Argabright, V., \& Hascall, P. 2011, Proc. SPIE, 8151,815117

*The community software is hosted at http://keplergo.arc.nasa.gov/Contributed Sof twarePyKEP.shtml.

$\dagger$ Cotrending basis vectors can be downloaded from http://archive.stsci.edu/kepler/ cbv.html. 